\title{
Sympathetic skin response-a method of assessing unmyelinated axon dysfunction in peripheral neuropathies
}

\author{
BHAGWAN T SHAHANI, JOHN J HALPERIN, ${ }^{*}$ PHILIPPE BOULU, $\dagger$ JEFFERY COHEN $\ddagger$
}

From the Laboratories of Clinical Neurophysiology and Neurocytology, Department of Neurology, Massachusetts General Hospital, Boston, Mass. USA

SUMMARY The sympathetic skin response (SSR) was measured in 33 patients with peripheral neuropathies and in 30 normal control subjects. Abnormalities of the response were correlated with clinical, pathologic, and EMG observations. The response was usually absent in axonal neuropathies, but present in demyelinating disorders. Abnormalities of the sympathetic skin response did not correlate well with clinical evidence of dysautonomia, but were a reliable indicator of disorders affecting unmyelinated axons.

In the study of disorders of the peripheral nervous system, non-invasive electrophysiologic tests have helped greatly in diagnosis and evaluation; morphologic methods have permitted more detailed study of biopsied nerve, and have helped to confirm the validity of newer electrophysiologic methods that assess the function of nerve fibres of different diameters. However, there remain inherent limitations of both morphologic and electrophysiologic methods. Conventional ultrastructural techniques are useful for identifying abnormal inclusions or deranged structure of organelles, ${ }^{2}$ but they do not discriminate among functional classes of axons. Morphometric methods detect abnormalities of unmyelinated fibres, but cannot differentiate autonomic from sensory axons. In contrast, electrophysiologic tests permit measurement of sensory and motor conductions in the same nerve, but study only the most rapidly conducting, heavily myelinated axons. These non-invasive tests, which evaluate nerve conduction velocity by either direct meas-

\footnotetext{
${ }^{*}$ Present address, Health Sciences Ctr, SUNY at Stony Brook, Stony Brook, NY.

†Present address, Hopital Beaujon, Clichy, France.

$\ddagger$ Present address, Mt Sinai Hospital Medical Center, New York, NY.

Address for reprint requests: Dr BT Shahani, Clinical Neurophysiology Laboratory, Massachusetts General Hospital, Fruit Street, Boston, MA 02114, USA.

Received 18 November 1983. Accepted 2 December 1983
}

urement or measurement of minimal late response latency, do not assess the state of slowly conducting myelinated or unmyelinated fibres.

Collision techniques ${ }^{3-6}$ help measure the function of the slower myelinated fibres, but cannot evaluate the function of unmyelinated fibres. Unmyelinated axons produce signals that are of such low amplitude and are so dispersed that they can only be well characterised by microneurography, which records directly from unmyelinated axons ${ }^{7-16}$ with intraneural needle electrodes. With this method it is even possible to differentiate between nerve fascicles mediating sympathetic control of the intramuscular vasculature (motor sympathetic activity (MSA)) and those conveying sympathetic impulses to the cutaneous blood vessels and sweat glands (skin sympathetic activity). ${ }^{72-16}$ (These two classes of sympathetic activity are apparently conveyed by separate fascicles of unmyelinated axons.) Unfortunately, microneurography is time-consuming, requires skilled, experienced operators, and is invasive, making routine use impractical.

However, the improved understanding of sympathetic activity that has been gained from microneurography has suggested a simple method for assessing autonomic function. Sympathetic skin activity mediates sudomotor function ${ }^{72} 15$ and a simple technique for assessing sudomotor function has been available for decades - the "galvanic skin response" (GSR). ${ }^{17} 18$ This change in skin resistance (which occurs after emotional or noxious stimuli, or most prominently, following deep inspiration) is triggered by increased sudomotor activity, mediated 
by the autonomic nervous system. Closely related to the GSR is the "sympathetic skin response", a change in the voltage measured from the surface of the skin, that is also attributable to sudomotor activity. The mechanism responsible for the sympathetic skin response is not known, but both the sympathetic skin response and the GSR seem to be mediated by the same afferent and efferent pathways.

Both sympathetic skin activity ${ }^{11}$ and the sympathetic skin response ${ }^{19}$ have been used in the past to evaluate autonomic function in patients with suspected dysautonomia. Both may be abnormal in such patients, but neither is invariably abnormal. In these patients these tests are most consistently abnormal when dysautonomia is associated with a severe peripheral neuropathy. ${ }^{19}$ This suggested to us that the sympathetic skin response might provide a simple way to test unmyelinated axon function in peripheral neuropathies. We therefore measured the sympathetic skin response in patients with neuropathies, and correlated the observations with clinical, EMG and, pathologic findings.

\section{Methods}

In the past 2 years we studied the sympathetic skin response in 33 patients being evaluated for peripheral neuropathy (ages 24 to 79 years, mean 56) (table 1). For some the test was requested by the referring physician because of the possibility of dysautonomia; in others, it was performed as part of the evaluation of suspected neuropathy. This report includes all patients who had the test performed during this period. To establish the normal range, we measured sympathetic skin responses in 30 normal adult volunteers (ages 13 to 62 years, mean 33), none of whom had any evidence of peripheral neuropathy or dysautonomia. In no subjects were there any adverse effects of the procedure.

Several factors alter the GSR and sympathetic skin response artefactually. The response habituates rapidly after repeated stimuli, ${ }^{17} 18$ so stimuli were delivered at irregular time intervals over an extended period of time. All tests were performed with the subject supine and relaxed in a semi-darkened room, with ambient temperature controlled at 22 to $24^{\circ} \mathrm{C}$. Since the response depends on body temperature, ${ }^{\circ}$ skin temperature was measured and if under $32^{\circ} \mathrm{C}$, the limbs were warmed. Body temperature (measured orally) was normal in all subjects at the time of the test.

The aim of the study was to devise a test of $\mathrm{C}$ fibre function that could be performed easily in clinical EMG laboratories. Therefore we did not perform other tests of autonomic function, or use the more technically demanding but standardised sympathetic skin response methods used in research laboratories studying the autonomic nervous system. ${ }^{21}$ Rather, we used standard surface EMG disc electrodes (TECA $10 \mathrm{~mm}$ diameter stainless steel surface electrodes), applied with commercial electrode paste to: (a) the palm and dorsum of the hand (b) the sole and
Table 1

\begin{tabular}{lcc}
\hline Diagnosis & $\begin{array}{l}\text { Pts with } \\
\text { sympathetic skin } \\
\text { response present }\end{array}$ & $\begin{array}{l}\text { Pts with } \\
\text { sympathetic skin } \\
\text { response absent }\end{array}$ \\
\hline Diabetes & 5 & 5 \\
Alcohol & 2 & 4 \\
Refsum's syndrome & 1 & \\
LGB & 2 & \\
MLD & 1 & \\
CMT & 1 & \\
LS plexopathy & 1 & 1 \\
Ischaemia & 1 & 1 \\
Hypoparathyroid & 1 & 2 \\
Paraneoplastic & 1 & 1 \\
$\begin{array}{l}\text { Neurodegenerative } \\
\text { Tabes dorsalis }\end{array}$ & & 2 \\
Undiagnosed & 2 & 16 \\
Totals & 17 & \\
\hline
\end{tabular}

dorsum of the foot (c) the anterior and posterior surfaces of the upper arm and (d) the patella and popliteal fossa. The band pass was 0.5-2000 Hz. In each patient, responses were measured in upper and lower extremities and the stimuli included the following: (a) Deep inspiration. To record the diaphragmatic EMG, two surface electrodes were placed $4 \mathrm{~cm}$ apart in the eighth intercostal space, with the anterior electrode in the anterior axillary line. (b) Electrical stimuli: Single square wave pulses of $0 \cdot 1 \mathrm{msc}$ duration, 10-20 mA intensity, were applied to the skin at the level of the wrist or ankle. Each type of stimulus was delivered 5-10 times.

Responses were recorded with standard EMG apparatus. The latency and amplitude of each response were measured manually. The latency was measured from the onset of the stimulus artefact (electrical stimulus) or diaphragmatic EMG activity to the beginning of the response; amplitude was measured peak to peak. Conduction velocity was estimated by recording simultaneously at two different sites (proximal and distal) in the same limb (electrode separation approximately $70 \mathrm{~cm}$ in the leg, $50 \mathrm{~cm}$ in the arm) following a single electrical stimulus and dividing the distance between the two recording sites by the difference in latency of the response at the two locations.

Histories were reviewed for symptoms of autonomic dysfunction (orthostatic dizziness or syncope, altered gastrointestinal function, or altered perspiration). Examinations included tests of postural changes in pulse and blood pressure, pulse response to Valsalva, qualitative assessment of skin temperature and sweating, and pupillary responses.

All patients had conventional motor and sensory conduction and late response studies, and EMG with concentric needle electrodes. In these tests, limb skin temperature was maintained at a minimum of $32^{\circ} \mathrm{C}$. Usual criteria were employed to divide patients into those with demyelinating neuropathies and those with axonal disease. Findings of (1) slowed conduction velocity (reduced to less than $60 \%$ of normal); (2) asynchronous, prolonged duration compound muscle action potential (CMAP); (3) prolonged distal latencies; (4) absence of sensory nerve action potentials; and (5) low persistence and prolonged latency of late responses suggested a primarily demyelinating process. Conversely, the presence of (1) reduced amplitude CMAP; 
(2) reduced amplitude compound nerve action potentials; (3) relatively normal conduction velocity; (4) mild prolongation (by less than $25 \%$ of normal) and normal persistence of late responses; and (5) abnormal spontaneous EMG activity (fibrillations and positive sharp waves) suggested an axonopathy.

In eight of the 33 patients, a sural nerve was biopsied. A $6 \mathrm{~cm}$ specimen was taken so that the distal end of the excised nerve was about $5 \mathrm{~cm}$ proximal to the lateral malleolus. Teased fibre preparations were performed on all biopsies. ${ }^{1}$ Portions of each biopsy were fixed in $1.25 \%$ glutaraldehyde, $1 \%$ paraformaldehyde, postfixed with osmium tetroxide, embedded in epon and sectioned for study by light and electron microscopy. Quantitative studies were performed on representative EMG photomontages, printed at a final magnification of 7000x. Data were collected using a Graf/pen digitiser, a PDP 11/23 computer, and software developed in our laboratory specifically for this purpose. Four nerves were studied with in vitro electrophysiologic methods. ${ }^{22}$

\section{Results}

\section{NORMAL CONTROLS}

The sympathetic skin response was present in all limbs of all normal subjects. In each subject the response amplitude varied from test to test, despite efforts to limit habituation. The shapes of the responses were similar in the arms and legs, but the amplitude (figs 1 and 2, table 2) was consistently greater in the hand than in the foot $(p<0.01$, Student's 2 tailed $t$ test) and was greater when triggered by deep inspiration than by electrical stimulation $(\mathrm{p}<0.01)$. The sympathetic skin response latency (fig 3) correlated well with the subject's height and was consistently longer in the leg than in the arm $(1.88 \pm 0.11$ vs $1.39 \pm 0.07$, mean $\pm S D, p<0.005$ Student's 1 tailed $t$ test). Estimates of unmyelinated
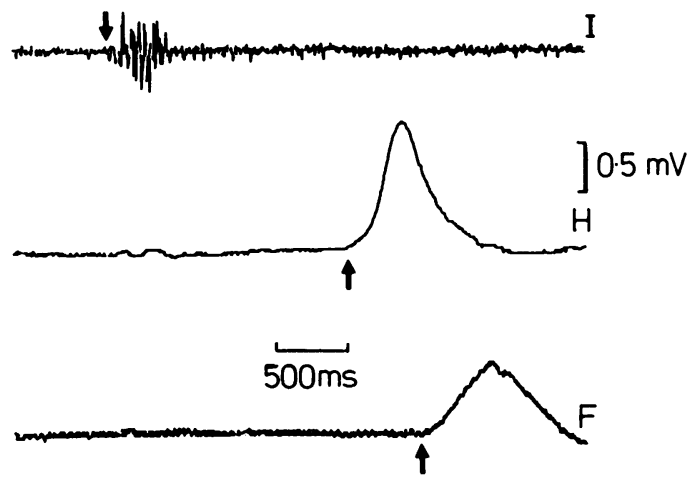

Fig 1 Example of the sympathetic skin response recorded simultaneously in the hand $(H)$ and foot $(F)$ of a control subject, following deep inspiration. Uppermost tracing is the diaphragmatic EMG activity. Upward pointing arrows indicate the onset of the response.

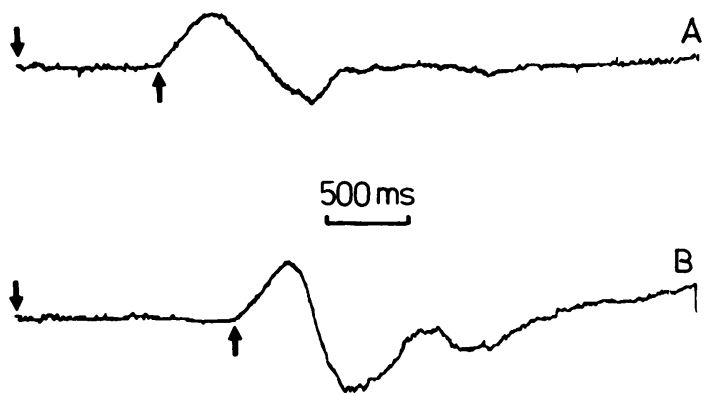

Fig 2 Sympathetic skin response recorded in the proximal arm $(A)$ and in the hand $(H)$ of the same limb, following an electrical stimulus. Downward pointing arrows indicate the stimulus, and upward pointing arrows demonstrate the onset of the response.

axon conduction velocity (five normal subjects) were $1.57 \pm 0.11$ in the arm and $1.02 \pm 0.07 \mathrm{~m} / \mathrm{s}$. in the leg $(\mathrm{p}<0.01,2$ tailed $t$ test) corresponding well with previous estimates from sympathetic skin activity measurements, ${ }^{101314}$ and with expectations for unmyelinated fibres.

\section{PATIENTS}

The sympathetic skin response was absent in 16 of the 33 patients. In one other patient a response was obtained in the hands but not the legs; in no other instance was a response seen in one limb but not others. In this same patient, a response was recorded after electrical stimulation but not after deep inspiration. In no other patient was a response elicitable by one class of stimulus but not by others. When responses were obtained, the sympathetic skin response amplitudes varied widely and did not differ significantly from controls (fig 3 ).

The presence or absence of sympathetic skin response did not correlate with symptoms suggesting dysautonomia. In only four of the 33 patients were

Table 2 Sympathetic skin response amplitude ( $\mathrm{mcV})$ :

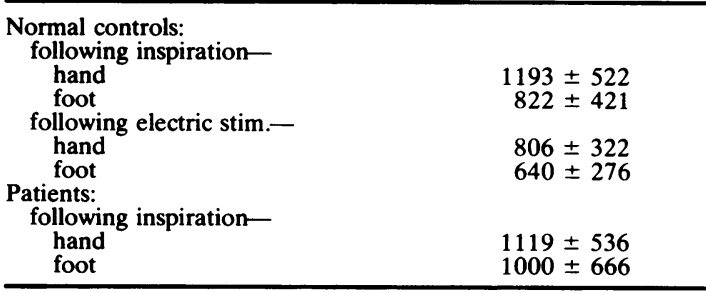

Sympathetic skin response amplitude (mean \pm standard deviation). In normal controls, the amplitude of the response in the hand is consistently greater than in the foot $(\mathrm{p}<0.01$. Student's 2 tailed $t$ test). In addition, in normals the amplitude following inspiration is greater than following an electrical stimulus ( $\mathrm{p}<0 \cdot 01,2$ tailed $t$ test). The difference between controls and patients is not significant. 


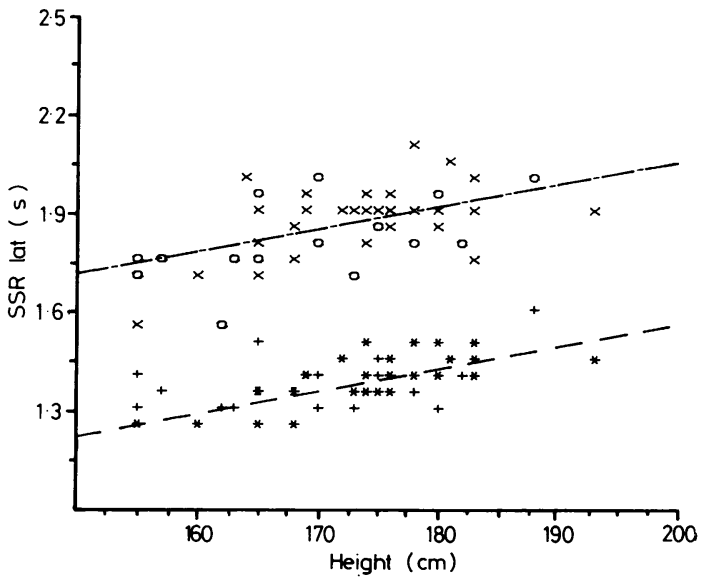

Fig 3 Sympathetic skin response latency following deep inspiration, as a function of height. Responses measured in the hand of normal subjects $\left({ }^{*}\right)$, the hand of those patients in whom a response was elicitable (+); the foot of control subjects $(X)$, and the foot of patients (O). The lower regression line ( $\rightarrow$ ) is a least squares fit to the latency in the hands of normal subjects. The upper regression line (. .) is a least squares fit to the latency in controls' feet.

there symptoms or physical findings suggestive of disordered autonomic function. In three patients these symptoms were prominent and sympathetic skin response was preserved. Of the 16 patients with no sympathetic skin response, only one had such symptoms. In contrast, absence of the sympathetic skin response did correlate with the "stockingglove" neuropathy (affecting all modalities equally)

Table 3

\begin{tabular}{|c|c|c|}
\hline & $\begin{array}{l}\text { Sympathetic skin } \\
\text { response present } \\
(n=17)\end{array}$ & $\begin{array}{l}\text { Sympathetic skin } \\
\text { response absent } \\
(n=16)\end{array}$ \\
\hline \multirow{2}{*}{$\begin{array}{l}\text { Sx of dysautonomia } \\
\text { EMG/NCT: }(n=33)^{*} \\
\text { primarily axonal } \\
\text { mixed } \\
\text { primarily demyelinating }\end{array}$} & 3 & 1 \\
\hline & $\begin{array}{l}5 \\
3 \\
9\end{array}$ & $\begin{array}{r}13 \\
3 \\
0\end{array}$ \\
\hline \multicolumn{3}{|l|}{$\begin{array}{l}\text { Pathology: }(n=8) \\
\text { teased fibre- }\end{array}$} \\
\hline $\begin{array}{l}\text { axonal } \\
\text { demyelinating } \\
\text { mixed }\end{array}$ & $\begin{array}{l}1 / 5 \\
3 / 5 \\
1 / 5\end{array}$ & $\begin{array}{l}1 / 3 \\
0 / 3 \\
2 / 3\end{array}$ \\
\hline \multicolumn{3}{|l|}{ quantitation- $\dagger$} \\
\hline & $2 / 5$ & $3 / 3$ \\
\hline $\begin{array}{c}\text { myelinated decreased } \\
\text { In vitro physiology }(n=4)\end{array}$ & $5 / 5$ & $3 / 3$ \\
\hline $\begin{array}{l}\text { A alpha decreased } \\
\text { A delta decreased } \\
\text { C decreased }\end{array}$ & $\begin{array}{l}4 / 4 \\
3 / 4 \\
0 / 4\end{array}$ & \\
\hline
\end{tabular}

*Correlation of absence of sympathetic skin response to axonal neuropathy significant at the $p<0.005$ level, Chi 2).

† Lower limits of normal, unmyelinated $28,000 / \mathrm{mm}^{2}$ of fascicle cross section, myelinated 7500 . Patients with decreased numbers of unmyelinated axons were all $<23,000$. seen in all but one of these 16 patients.

All patients had prolonged or absent responses. Two had normal sensory conductions; three had normal motor conductions. Of the 17 patients with preserved sympathetic skin response, nine had electrophysiologic findings of a demyelinating neuropathy; in five the tests implied an axonal process. In three, the findings suggested a mixed demyelinating and axonal disorder (table 3 ). In contrast, 13 of the 16 patients with no sympathetic skin response had abnormalities consistent with an axonopathy; in the other three, the tests suggested a mixed process.

These electrophysiologic findings suggested that absence of sympathetic skin response was associated with axonal disorders. To test this statistically, it was necessary first to consider those patients who had findings suggestive of disorders affecting both axons and myelin. We grouped these patients in a manner that would bias the conclusions against our hypothesis. In patients with preserved sympathetic skin response, we considered those with mixed findings to have an axonal process. In those with no sympathetic skin response, we combined the patients with mixed abnormalities with those with demyelinating disorders. Despite this grouping, the relationship was still significant $(p<0.01, \mathrm{Chi})$.

Of the eight patients with sural nerve biopsies, the

\section{Axons/sq mm $(\times 1000)$}

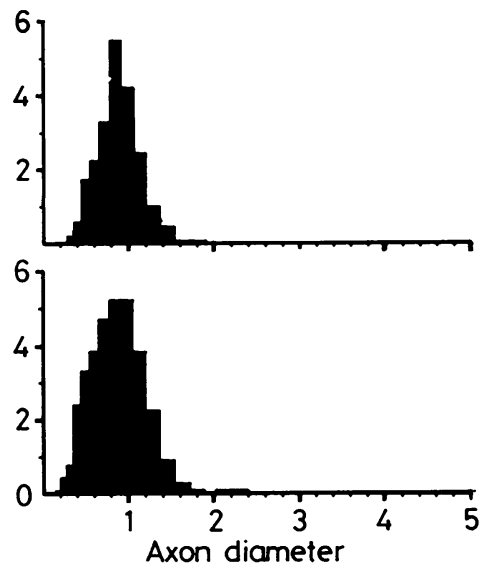

Fig 4 Distribution of unmyelinated axon size. In each histogram, axon diameter is plotted on the $X$ axis. The number on the $Y$ axis is, for each diameter range, the number of axons per $\mathrm{mm}$ of nerve fascicle cross section. The upper graph is of a biopsy from a patient in whom the sympathetic skin response was absent. The overall density (axons/mm) of unmyelinated axons was 21,900, (abnormally low). The lower graph is of a biopsy from a patient in'whom the sympathetic skin response was preserved. The overall density of unmyelinated axons was 33,400 (normal). 

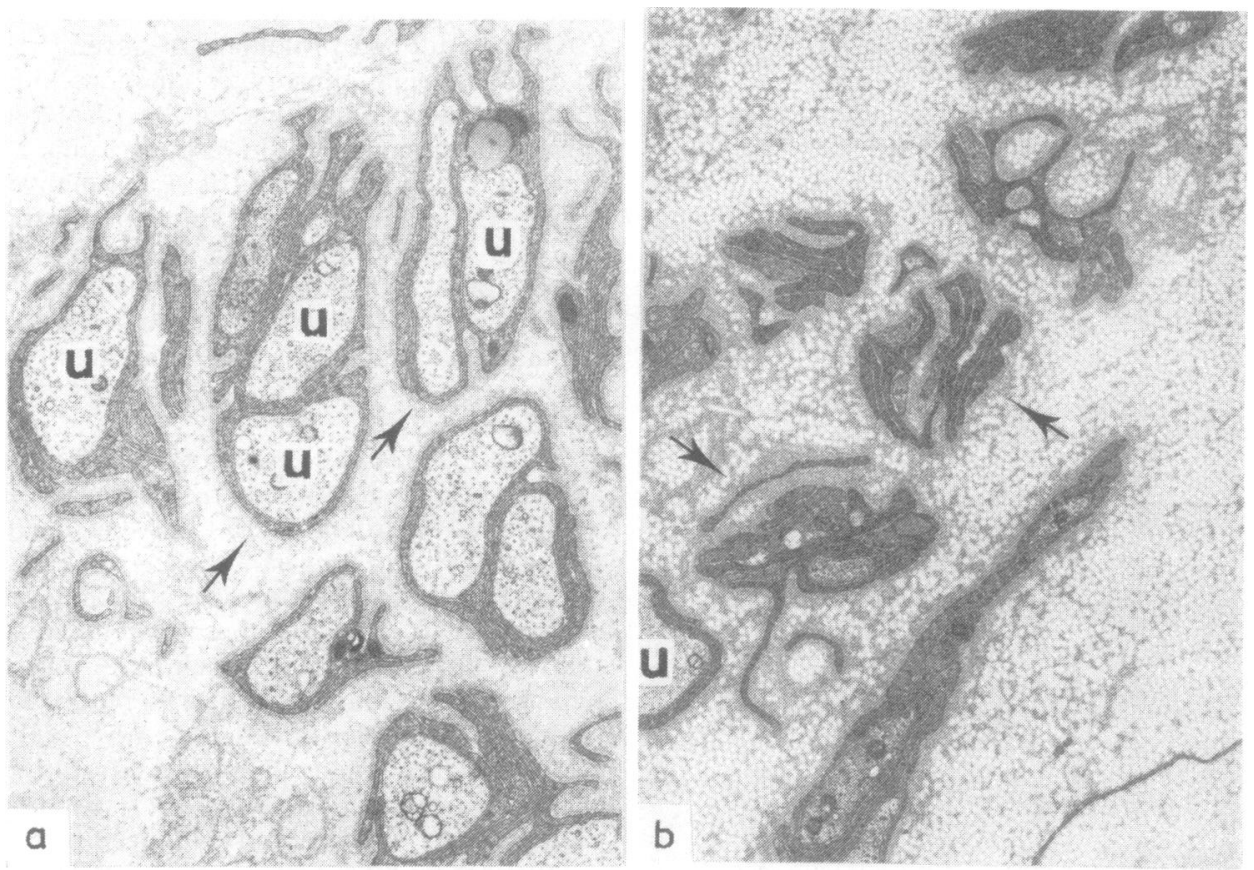

Fig 5 Electron micrographs of representative regions of nerve fascicles from a patient with the sympathetic skin response present (a) (unmyelinated axon density 31,900 axons/mm) and sympathetic skin response absent (b) (unmyelinated axon density 17,100). In (a) most Schwann cell processes (arrows) enclose unmyelinated axons $(u)$. In (b) most Schwann cell processes (arrows) do not envelope axons, but instead wrap around each other or around bundles of collagen fibrils. In $(b)$ the amount of endoneurial collagen is increased. Only three unmyelinated axons $(u)$ are seen in $(b)$ compared to twelve in $(a)$.

(Magnification $\times 12$ 000).

sympathetic skin response was absent in three. Of the five with preserved sympathetic skin response, teased fibre preparations demonstrated segmental demyelination in three, predominantly segmental demyelination but with some evidence of an axonopathy in one, and primarily axonal degeneration in one. This last patient had an unusual clinical picture suggesting a patchy lumbosacral plexopathy that spared some parts of the nerve while affecting others (presumably sparing enough axons to preserve the reflex). Of the three patients with no sympathetic skin response, teased fibre preparations were consistent with an axonopathy in all three although two also had some evidence of mild segmental demyelination (commensurate with that described in some axonopathies.) $)^{23}$ In four biopsied patients with sympathetic skin response, in vitro electrophysiologic testing was performed. In all, C fibre function was preserved while $A$ alpha and $A$ delta functions were markedly decreased.

Quantitative morphometric studies confirmed that the absent sympathetic skin response correlated best with disorders of unmyelinated axons (figs 4 and 5). All three patients with absent sympathetic skin response had decreased numbers of unmyelinated fibres $(17-22,000 / \mathrm{m})$ (table 3$)$. Of the five patients with preserved sympathetic skin response, three had normal numbers of unmyelinated fibres and significantly decreased numbers of myelinated axons. Of the other two, one patient had an unique multisystem degenerative disorder, decreased numbers of myelinated and unmyelinated fibres (18 500), and symptoms of dysautonomia. In this patient, there was prominent segmental demyelination, and the decreased number of unmyelinated fibres probably reflected an increase in endoneurial connective tissue (decreasing the number of axons $/ \mathrm{mm}$ of fascicle cross section). The last patient was the woman with a patchy plexopathy, with decreased numbers of both myelinated and unmyelinated axons $(18000)$ in some fascicles but not others. It is likely that more proximally, some autonomic trunks were partially spared by such a process, preserving the sympathetic skin response.

The very small sample size precluded a valid statistical comparison of these two groups. How- 
ever, the pathologic and in vitro physiologic findings (table 3) qualitatively support the conclusions based on EMGs. The sympathetic skin response was absent in patients with axonopathies that severely affected unmyelinated axons, and was preserved when these fibres were spared. The pathologic findings correlated better with the presence or absence of sympathetic skin response than with clinical evidence of dysautonomia. Of the five biopsied patients with sympathetic skin response present and pathology suggesting a demyelinating or mixed process, three had prominent symptoms of disordered sympathetic and para-sympathetic function. Two of these had decreased numbers of unmyelinated fibres in the sural nerve, one did not. Of the three biopsied patients with no sympathetic skin response, none had symptoms of disordered autonomic function, and all had decreased numbers of unmyelinated axons.

\section{Discussion}

The sympathetic skin response was readily elicitable in normal subjects. With double recording techniques, it was possible to derive an estimate of conduction velocity for unmyelinated axons, which agreed with that derived by microneurography in sudomotor fibres. ${ }^{11}$ In patients with neuropathies, the response amplitude and latency remained in the normal range, so long as the sympathetic skin response was present. Therefore the sympathetic skin response could only be considered definitely abnormal when absent. Using this criterion alone, we could subdivide our patients into two groups those with and those without sympathetic skin response. (A third sub-group consisted of one patient who had a response in one limb but not others. This observation suggests that the technique could be used to assess isolated lesions of unmyelinated axons, but this has not yet been pursued further.) When we then subdivided patients into those with electrophysiologic evidence of axonopathies and those with demyelination, axonal disorders correlated strongly with absence of sympathetic skin response. Conversely, those with predominantly demyelination had intact sympathetic skin responses. These findings were substantiated by morphologic findings in sural nerve biopsies. Absence of sympathetic skin response correlated best with disorders that preferentially affected unmyelinated axons.

Abnormalities of sympathetic skin response did not correlate with clinical evidence of dysautonomia. In previous studies of sympathetic skin activity and sympathetic skin response, the correlation between abnormalities of these tests and dysautonomia was weak. In contrast, most studies found a relationship between abnormalities of these tests and abnormal autonomic function when the latter was associated with a severe peripheral neuropathy. ${ }^{119}$ This lack of correlation between clinical evidence of dysautonomia and abnormalities of this test of sympathetic function initially seems surprising. However, the sympathetic skin response specifically tests the skin sympathetic fibre-not the parasympathetic fibres or the motor sympathetic fibres, that mediate many of the clinical symptoms of dysautonomia. It is a common clinical observation that sudomotor function is disordered in peripheral neuropathies, such as diabetic neuropathy, ${ }^{24}$ even when there is no evidence of other abnormalities of autonomic function. It seems likely that a diffuse, "dying back" type of peripheral neuropathy would affect many small, distal sudomotor fibres and thereby alter sympathetic skin activity long before other sympathetic or parasympathetic fibres were damaged sufficiently to cause orthostatic hypotension or other signs of dysautonomia. For this reason, sympathetic skin response is a better marker of peripheral neuropathies than of dysautonomia. We then conclude that the sympathetic skin responseas performed in this study-can document axonal pathology, especially when unmyelinated axons are involved, but does not assess autonomic function.

Three recent studies ${ }^{25-27}$ have suggested that the sural nerve contains few sympathetic fibres. Our observations bear on this data in two ways. First, in one of the three biopsied patients with prominent symptoms of autonomic nervous system dysfunction, there were no abnormalities of unmyelinated axons in the sural nerve, as also observed in some $^{2829}$ but not all ${ }^{3031}$ previously published reports of sural nerve biopsies in dysautonomia. In contrast, three patients with marked abnormalities of unmyelinated axons in the sural nerve had no symptoms of dysautonomia. We take this as further evidence that, in a broader sample of patients than reported in previous studies, the sural nerve probably does not contain many sympathetic fibres. On the other hand, we noted the frequent occurrence of both abnormalities of unmyelinated axons in the sural nerve, and absence of the sympathetic skin response. Therefore the sympathetic skin response is absent in diseases in which small unmyelinated axons of many types - sensory and autonomic - are damaged.

The sympathetic skin response can be recorded with commonly available EMG equipment. It may be present when conventional motor and sensory conduction and late response studies demonstrate gross abnormalities, or absent when these tests are normal. These findings suggest that recording the 
sympathetic skin response is a new and useful method of evaluating a part of the peripheral nervous system-small unmyelinated $C$ fibres - that cannot be assessed by the studies currently performed in clinical EMG laboratories.

The authors gratefully acknowledge the technical assistance of Mr L Cherkas and Mrs H Goolsby. This work was supported in part by NIH postdoctoral fellowship NS06638 (Dr J Halperin). Sympathetic skin response in peripheral neuropathies.

\section{References}

' Asbury AK, Johnson PC. Pathology of Peripheral Nerve. Philadelphia: W. B. Saunders, 1978.

${ }^{2}$ Ochoa J. Recognition of unmyelinated fibre disease: morphologic criteria. Muscle Nerve 1978;1:375-87.

${ }^{3}$ Domingue J, Shahani BT, Young RR. In vivo documentation of dysfunction in different diameter alpha motor axons. Trans Am Neurol Assoc 1980;105:437-9.

${ }^{4}$ Douglas WW, Ritchie JM. A technique for recording functional activity in specific groups of medullated and non-medullated fibres in whole nerve trunks. J Physiol (Lond) 1957;138:19-30.

${ }^{5}$ Hopf HC. Untersuchungen uber die Unterschiede in der Leitgeschwindigkeit motorischer Nervenfasern beim Menschen. Deutsch Z Nervenheilk 1962;183:579-88.

- Shahani BT, Potts F, Juguilon A, Young RR. Maximal-minimal motor nerve conduction and $F$ response studies in normal subjects and patients with ulnar nerve compression. Muscle Nerve 1980;3:182.

${ }^{7}$ Bini G, Hagbarth K-E, Hynninen P, Wallin BG. Thermoregulatory and rhythm-generating mechanisms governing the sudomotor and vasoconstrictor outflow in human cutaneous nerves. $J$ Physiol (Lond) 1980;306:537-52.

${ }^{8}$ Delius W, Hagbarth K-E, Hongell A, Wallin BG. Manoeuvres affecting sympathetic outflow in human muscle nerves. Acta Physiol Scand 1972;84:82-94.

9 Delius W, Hagbarth K-E, Hongell A, Wallin BG. Manoeuvres affecting sympathetic outflow in human skin nerves. Acta Physiol Scand 1972;84:177-86.

${ }^{10}$ Fagius J, Wallin BG. Sympathetic reflex latencies and conduction velocities in normal man. $J$ Neurol $S c i$ 1980;47:433-48.

"Fagius J, Wallin BG. Sympathetic reflex latencies and conduction velocities in patients with polyneuropathy. J Neurol Sci 1980;47:449-61.

${ }^{12}$ Hagbarth K-E, Hallin RG, Hongell A, et al. General characteristics of sympathetic activity in human skin nerves. Acta Physiol Scand 1972;84:164-76.

${ }^{13}$ Hallin RG, Torebjork HE. Single unit activity in human skin nerves during rest and various manoeuvres. Acta Physiol Scand 1974;92:303-17.

${ }^{14}$ Hallin RG, Torebjork HE. Methods to differentiate electrically induced afferent and sympathetic $\mathrm{C}$ unit responses in human cutaneous nerves. Acta Physiol Scand 1974;92:318-31.

${ }^{15}$ Torebjork HE. Afferent $\mathrm{C}$ units responding to mechanical, thermal and chemical stimuli in human nonglabrous skin. Acta Physiol Scand 1974;92:374-90.

${ }^{16}$ Vallbo AB, Hagbarth K-E, Torebjork HE, Wallin BG. Somatosensory, proprioceptive, and sympathetic activity in human peripheral nerves. Physiol Rev 1979;59:919-57.

${ }^{17}$ Lader MH, Montagu JD. The psycho-galvanic reflex: a pharmacologic study of the peripheral mechanism. $J$ Neurol Neurosurg Psychiatry 1962;25:126-33.

${ }_{18}$ Prout BJ. Independence of the galvanic skin reflex from the vasoconstrictor reflex in man. $J$ Neurol Neurosurg Psychiatry 1967;30:319-24.

${ }^{19}$ Goadby HK, Downman CBB. Peripheral vascular and sweat-gland reflexes in diabetic neuropathy. Clin Sci Mol Med 1973;45:281-9.

${ }^{20}$ Franz DN, Iggo A. Conduction failure in myelinated and non-myelinated axons at low temperatures. $J$ Physiol (Lond) 1968;199:319-45.

${ }^{21}$ Martin I, Venables PH, eds. Techniques in Psychophysiology. New York: John Wiley and Sons, 1980.

${ }^{22}$ Adams RD, Shahani BT, Young RR. A severe pansensory polyneuropathy. Trans Am Neurol Assoc 1973;98:13-5.

${ }^{23}$ Dyck PJ, Johnson WJ, Lambert EH, O'Brien PC. Segmental demyelination secondary to axonal degeneration in uremic neuropathy. Mayo Clin Proc 1971;46:400-31.

${ }^{24}$ Thomas PK. Diabetic neuropathy. In: Dyck PJ, Thomas PK, Lambert EH, eds. Peripheral Neuropathy Philadelphia: WB Saunders 1975.

${ }^{25}$ Chad D, Shoukimas GM, Bradley WG et al. Peripheral nerve unmyelinated axons following lumbar sympathectomy. Ann Neurol 1981;10:486-8.

${ }^{26}$ Galassi G, Nemni R, Baraldi A et al. Peripheral neuropathy in multiple system atrophy with autonomic failure. Neurol (NY) 1982;32:1116-21.

${ }^{27}$ Sjo O, Buchthal F, Henriksen O, Segrsen P. The absence of fibres in the sural nerve mediating vasoconstriction. Acta Physiol Scand 1976;98:379-80.

${ }^{28}$ Appenzeller O, Kornfield M. Acute pandysautonomia. Arch Neurol 1973;29:334-9.

${ }^{29}$ Young RR, Asbury AK, Corbett JL, Adams RD. Pure pan-dysautonomia with recovery. Brain 1975;98:613-36.

${ }^{30}$ Low PA, Dyck PJ, Lambert EH et al. Acute pandysautonomia. Ann Neurol 1982;12:78.

${ }^{31}$ Low OA, Dyck PJ, Lambert EH, Brimijoin WS, Trautman JC, Malageda JR, Fealy RD, Barrett DM. Acute panautonomic neuropathy. Ann Neurol 1983;13:412-7. 